\title{
Total intravenous anaesthesia- Target Controlled Infusion and Superimposed High-Frequency Jet Ventilation - an anaesthetic protocol for interventional bronchoscopy in cancer patients
}

\author{
Irina Ristescu ${ }^{*}, 1,2$, Iulia Jitaru ${ }^{1}$, Alexandru Parfeni², Corina Dascălu², Beatrice \\ Cobzaru $^{2}$, loana Grigoraș ${ }^{1,2}$
}

${ }^{1}$ Anesthesia and Intensive Care Department, Regional Institute of Oncology lasi, Romania, ${ }^{2}$ "Grigore T. Popa" University of Medicine and Pharmacy, Iaşi, Romania;

\begin{abstract}
Introduction: Diagnostic or therapeutic bronchoscopy may be performed under general anaesthesia in special circumstances aiming for patient safety and operator comfort. Tracheobronchial instrumentation requires an open system ventilatory support. We report the first case series of oncologic patients managed with Total Intravenous Anaesthesia-Target Controlled Infusion (TIVA-TCI) and Superimposed High-Frequency Jet Ventilation (SHFJV) during flexible and rigid bronchoscopy in our institution. We evaluated the safety profile of this technique in cancer patients. Methods: We analysed 24 oncological patients requiring bronchoscopy under general anaesthesia during a 9 months period. The anaesthetic protocol consisted in TIVA-TCI with propofol, Schnider model, site effect concentration, with standard intra-anaesthetic monitoring plus hypnosis and neuromuscular function monitoring and SHFJV using Twin Stream ${ }^{\mathrm{TM}}$ ventilator. Results: A number of 25 interventions were performed - 19 flexible and 6 rigid bronchoscopy. During the procedures, optimal anaesthesia depth (bispectral index=40-60) was maintained with a mean propofol site effect concentration $\mathrm{Ce}=3.1(+/-0.6)$ $\mu \mathrm{g} / \mathrm{ml}$. Hemodynamic parameters varied $+/-10-20 \%$ of preoperative values. $\mathrm{SpO}_{2}$ was stable with a mean value of $98.5 \%\left(\mathrm{FiO}_{2}=0.5-1\right)$ and intermittently measured etCO $\mathrm{C}_{2}$ had a mean value of $32(+/-5.4) \mathrm{mmHg}$. Airway pressures varied according to procedural particularities with a safety upper limit of $25 \mathrm{mmHg}$. We recorded no intra-anaesthetic or post-interventional complications. Conclusion: The anaesthetic protocol including TIVA-TCI and SHFJV is a safe and effective method of maintaining adequate depth of anaesthesia, cardiovascular stability and gas exchange during diagnostic or therapeutic bronchoscopy in cancer patients.
\end{abstract}

Keywords: superimposed high frequency ventilation, TIVA-TCI, cancer patients, interventional bronchoscopy.

\section{Introduction}

The diagnostic and/or therapeutic tracheobronchial instrumentation consisting of

Received: August 2015; Accepted after review: September 2015; Published: September 2015.

${ }^{*}$ Corresponding author: Irina Ristescu, MD, PhD, "Grigore T. Popa" University of Medicine and Pharmacy lasi, Anesthesia and Intensive Care Department, Regional Institute of Oncology laşi, 2-4, Gen. Henri Mathias Berthelot St., 700483 laşi, Romania

Email: iristescu@yahoo.com flexible or rigid bronchoscopy can be an unpleasant, painful or technically difficult procedure. In order to ensure patient safety and operator comfort some of these procedures must be performed under general anaesthesia.

Providing an adequate oxygenation and ventilation during these interventions can be sometimes challenging, even in patients with normal lung function, due to the concomitant manipulation of the airway. Historically, 
several methods of ventilation have been used: apneic oxygenation, spontaneous assisted ventilation, controlled ventilation in a closed system, manual jet ventilation and highfrequency jet ventilation (HFJV) [1]. Jet ventilation techniques, defined as the injection of a gas through a small tube using a high pressure gas source (1-3 bar), are now increasingly applied in ENT surgery, pneumology, thoracic surgery and intensive care. Two modes of jet ventilation are currently available. The manual jet ventilation was originally described in 1967 [2] and has the advantage of a low cost equipment. It is now replaced by HFJV, an automated ventilatory support largely used to facilitate airway procedures [3]. In both types of jet ventilation complications as hypercapnia, barotrauma or hypoxia may occur $[4,5]$.

Superimposed High-Frequency Jet Ventilation (SHFJV) is a newly developed technique consisting in a combination of two jet streams - a pulsatile high-frequency jet ventilation (HF) $(50-1500 / \mathrm{min})$ and a lownormal frequency jet ventilation (NF) (12$20 / \mathrm{min}$ ) that can be separately or simultaneously applied [6]. The ventilation in this open system results from the combination of set driving pressure, frequency and inspiratory/expiratory ratio. The high frequency jet ventilation generates an expiratory plateau pressure creating a positive end expiratory pressure responsible for the oxygenation through diffusion mechanisms. The normal frequency ventilation jet creates an inspiratory plateau pressure which is responsible for the elimination of $\mathrm{CO}_{2}$ through convection mechanism [7].

Challenges to perform safe general anaesthesia in cancer patients with multiple comorbidities and often marginal pulmonary function include: limited availability of specialized equipment, the need for additional training and lack of experience with uncommon techniques, and the need for rapid onset/offset of the anaesthetic effects.

In the present study we report our initial experience using TIVA-TCI and SHFJV in diagnostic and interventional bronchoscopy in a case series of cancer patients. We analysed the safety profile of this anaesthetic protocol developed for these interventions.

\section{Methods}

\section{Patient characteristics}

All 24 consecutive patients proposed for flexible/rigid bronchoscopy under general anaesthesia were analysed. Patients were enrolled in the first 9 months of SHFJV use in our institution. The majority of patients had suspected or confirmed lung cancer and they were scheduled for diagnostic or staging bronchoscopy.

Patient characteristics, diagnosis and types of the procedures are listed in Tables 1, 2,3 respectively.

Table 1. Patient data

\begin{tabular}{cc}
\hline & All patients $(\mathbf{n}=\mathbf{2 4})$ \\
\hline Age $(\mathbf{y r})$ & $58.2( \pm 10.2)^{*}$ \\
\hline Sex ratio M/F & $17 / 7$ \\
Weight $(\mathbf{k g})$ & $67( \pm 11)^{*}$ \\
\hline Height $(\mathbf{c m})$ & $167( \pm 8.3)^{*}$ \\
\hline ASA $^{* *}$ status $(\mathbf{I I} / \mathrm{III})$ & $16 / 8$ \\
\hline & \\
& ${ }^{*}$ mean $( \pm \mathrm{SD}),{ }^{* *}$ American Society of Anesthesiologists
\end{tabular}


Table 2. Diagnosis of patients undergoing bronchoscopy under SHFJV

\begin{tabular}{cc}
\hline Diagnosis & Total $(\mathbf{n}=\mathbf{2 4})$ \\
\hline Lung cancer & 19 \\
\hline Hodgkin disease & 2 \\
Mediastinal nodes & 2 \\
Foreign bodies & 1 \\
\hline
\end{tabular}

Table 3. Technique and endoscopic intervention performed under SHFJV

\begin{tabular}{lcc}
\hline \multicolumn{1}{c}{ Technique } & Intervention & Total $(\mathbf{n}=\mathbf{2 5})$ \\
\hline Flexible bronchoscopy & EBUS TBNA ${ }^{*}$ diagnosis & 19 \\
\hline Rigid bronchoscopy & Tumoral biopsy & 3 \\
& Intrabronchial glue instillation for fistula closure & 2 \\
\hline
\end{tabular}

*EBUS TBNA - Endobronchial ultrasound-guided transbronchial needle aspiration

Mean duration of general anaesthesia for endoscopic intervention was $68(+/-16.6)$ minutes.

The following parameters were recorded in each patient: hemodynamics heart rate (HR), blood pressure (BP), oxygenation - $\mathrm{SpO}_{2}$, hypnosis - bispectral index (BIS), ventilation - etCO ${ }_{2}$, airway pressures and muscle relaxation - TOF ratio. We analysed the recorded data measured before, during (every 5 minute) and after the procedure.

\section{Anaesthetic protocol}

We used a standardized general anaesthesia consisting of preoperative assessment, propofol TIVA-TCI, effect site concentration, fentanyl and rocuronium, standard, BIS and TOF monitoring and open system ventilation with SHFJV. The emergence consisted of rocuronium reversal (sugammadex) and interruption of propofol TCl.

\section{Preoperative assessment}

Preoperative airway assessment was focused on teeth mobility, interdental distance and neck range of motion. Full blood count, glycaemia, electrolytes, renal function, coagulation tests, chest imaging (CT) and electrocardiogram were performed before the intervention. Contraindications for the procedure were acute respiratory failure, significant arrhythmias, decompensated heart failure and high bleeding risk. All patients followed the European Society of Anesthesiology preoperative fasting protocol [8].

\section{Anaesthetic technique}

After three minutes preoxygenation with $\mathrm{FiO}_{2}=1$, anaesthesia was induced with fentanyl 1-5 $\mu \mathrm{g} / \mathrm{kg}$, lidocaine $1 \mathrm{mg} / \mathrm{kg}$, propofol - TCl, Schnider model, setting a variable site effect concentration (Ce) between 4.0 and $6.0 \mu \mathrm{g} / \mathrm{ml}$ based on BIS values, and rocuronium bromide $0.6 \mathrm{mg} / \mathrm{kg}$. The patients were ventilated using 
face mask and close circuit untill TOF value reached two twitches, followed by the insertion of rigid bronchoscope or a laryngeal mask in case of flexible bronchoscopy. Anaesthesia was maintained with propofol TCl Ce between $2.5-4 \mu \mathrm{g} / \mathrm{ml}$, in order to maintain an adequate hypnosis (BIS=40-60). fentanyl $2 \mu \mathrm{g} / \mathrm{kg}$ and rocuronium bromide $0.2 \mathrm{mg} / \mathrm{kg}$ were given as required. sugammadex, was used to reverse rocuronium at the end of the procedure. The dose was adjusted to the level of neuromuscular blockade -2 or $4 \mathrm{mg} / \mathrm{kg}$.

\section{Ventilation technique}

Jet ventilation was performed with Twin Stream ${ }^{\mathrm{TM}}$ (Carl Reiner $\mathrm{GmbH}$, Vienna, Austria) jet ventilator (Figure 1) containing two separately or simultaneously functioning ventilation units: high frequency (HF) ventilator and normal frequency (NF) ventilator (Figure 2).

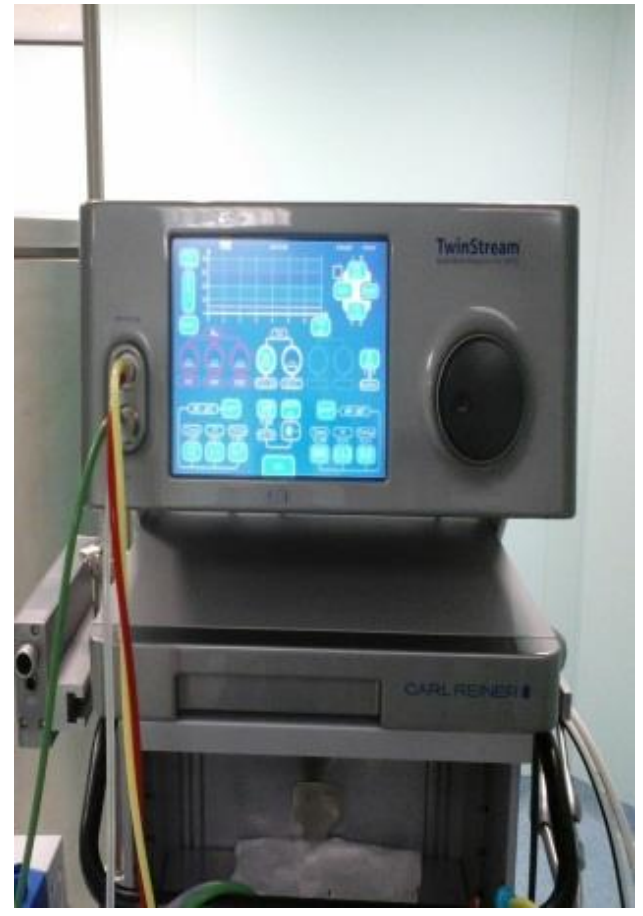

Fig. 1.Twin Stream jet ventilator

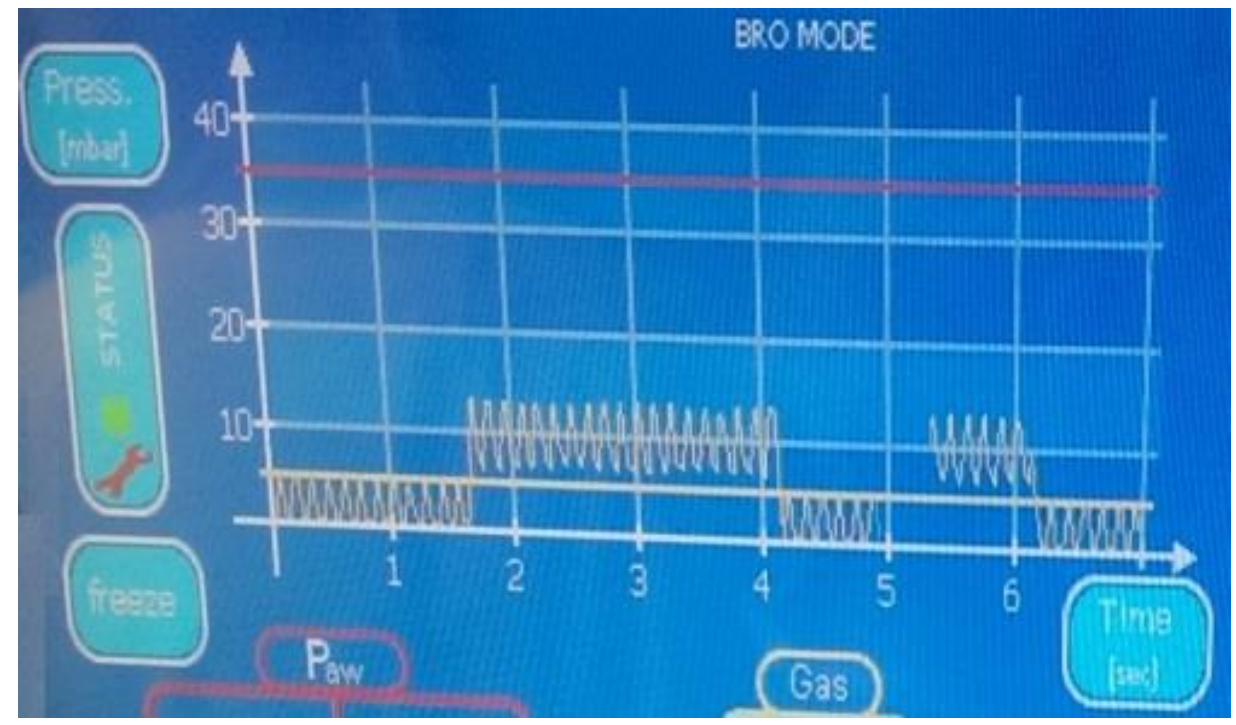

Fig. 2. High frequency and normal frequency jet ventilation

For flexible bronchoscopy procedures endobronchial ultrasound transbronchial needle aspiration (EBUS TBNA) - a laryngeal mask (LMA) was inserted after the induction of anesthesia. The proper position of LMA was initially checked with closed ventilation circuit.
A Jet-Converter (Carl Reiner GmbH, Vienna, Austria) was attached to the LMA for supraglottic jet ventilation (Figure 3). In patients with rigid bronchoscopy trachea was intubated with a Jet-Bronchoscope (Carl Reiner $\mathrm{GmbH}$, Vienna, Austria). 


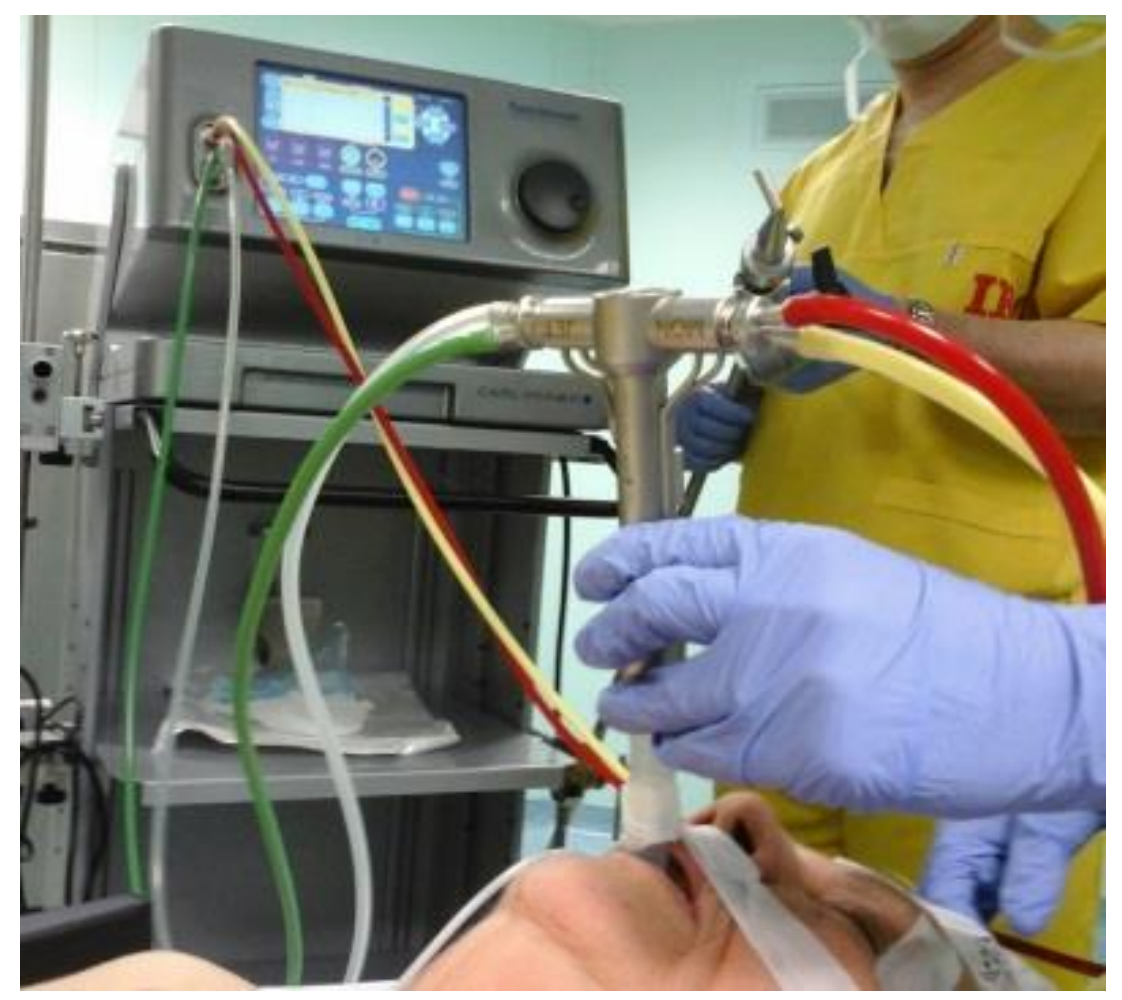

Fig. 3. Jet-Converter attached to the laryngeal mask

Four lumen catheters bronchoscopy (BRO) mode was used in all patients. Two ventilation catheters - HF (green) and NF (white) - and two monitoring catheters - airway pressures (red) - peak inspiratory pressure (PIP), mean airway pressure (MAP), positive- end expiratory pressure (PEEP) and gas analysis (yellow) - inspiratory oxygen concentration $\left(\mathrm{FiO}_{2}\right.$ jet), the ventilated patient's oxygen concentration $\left(\mathrm{FIO}_{2} \mathrm{AW}\right)$, intermittent end tidal $\mathrm{CO}_{2}\left(\right.$ etCO $\left.\mathrm{C}_{2}\right)$, were laterally attached to the convertor (Figure 4).

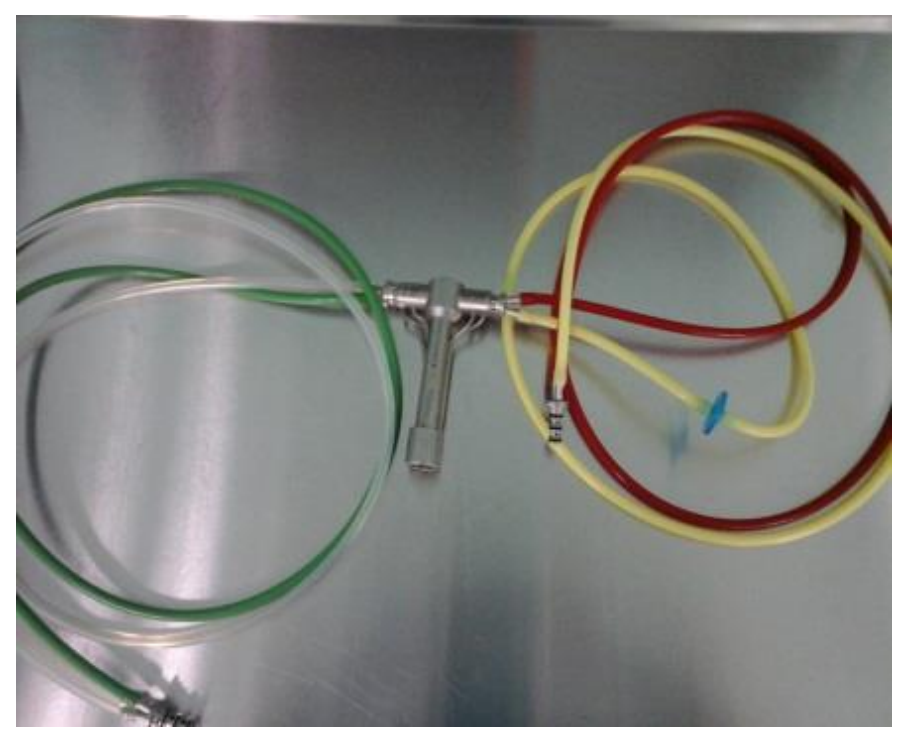

Fig. 4. The four catheters attached to the convertor 
After choosing the BRO mode, the ventilator automatically displays set parameters based on a body weight algorithm. These parameters are: for HF ventilator emission pressure - $P_{H F[b a r]}=0.1-2$ bar, ventilation frequency $-f[1 / \mathrm{min}]=50-1500 / \mathrm{min}$, inspiratory/expiratory ratio $\mathrm{I}: \mathrm{E}=1: 5-3: 1$ and for $\mathrm{NF}$ ventilator emission pressure $-\mathrm{P}_{\mathrm{NF}[\mathrm{bar}]}=0.1$ 3.5 bar, ventilation frequency $-f[1 / \mathrm{min}]=1$ $100 / \mathrm{min}$, inspiratory/expiratory ratio $\mathrm{I}: \mathrm{E}=1: 5$ 3:1 (Figure 5).

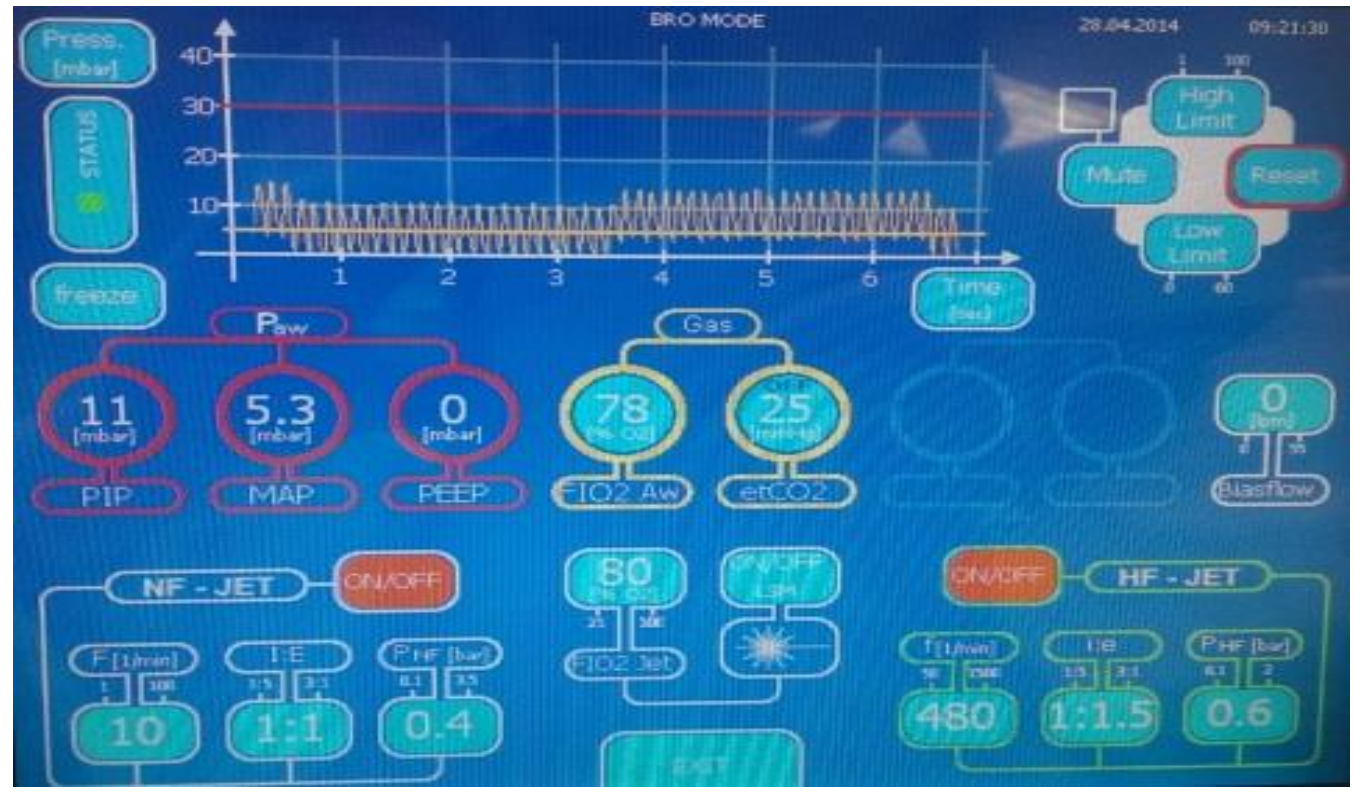

Fig. 5. Automatically selected parameters in bronchoscopy mode

Subsequently, these parameters can be adjusted in order to achieve an optimal oxygenation and ventilation.

The TwinStream jet ventilator allows intermitent et $\mathrm{CO}_{2}$ measurement. By selecting etCO ${ }_{2}$ icon on the main screen, $\mathrm{HF}$ stops automatically, NF runs for five cycles and, after etCO $\mathrm{C}_{2}$ assessment, both types of JV start again.

We set a safety upper limit for the peak airway pressure of $25 \mathrm{mmHg}$.

When the procedure was completed the neuromuscular block was reversed with sugammadex (dose dependent on TOF values), propofol $\mathrm{TCl}$ was stopped and the jet ventilator was switched off. The patients were ventilated by face mask or LMA using the circle circuit till adequate spontaneous breathing.

\section{Intra-anaesthetic monitoring}

The following intra-anaesthetic monitoring (Infinity C700 Draeger) was used in all patients:

- hemodynamic monitoring - ECG 2 leads (DII, V4), heart rate (HR), continuous ST segment monitoring, noninvasive blood pressure (BP)

- oxygenation monitoring - peripheral oxygen saturation $\left(\mathrm{SpO}_{2}\right)$

- hypnosis monitoring - bispectral index (BIS)

- neuromuscular function monitoring train of four (TOF).

Ventilator monitoring (Twin Stream, Carl Reiner $\mathrm{GmbH}$ ) consisted of peak inspiratory pressure (PIP), mean airway pressure (MAP), positive-end expiratory pressure (PEEP), inspiratory oxygen concentration $\left(\mathrm{FiO}_{2}\right.$ jet), the ventilated patient's oxygen concentration $\left(\mathrm{FIO}_{2}\right.$ $\mathrm{AW})$, intermittent end tidal $\mathrm{CO}_{2}\left(\mathrm{etCO}_{2}\right)$. 
After complete recovery all patients were transferred to Post Anaesthesia Care Unit.

All recorded parameters are presented as mean values $+/$-standard deviation.

\section{Results and discussion}

In the present study we report our first case series of cancer patients scheduled for diagnostic and interventional bronchoscopy managed with TIVA-TCI and SHFJV. The procedures consisted in flexible and rigid bronchoscopy, the majority of our patients ( $n=19 / 24)$ undergoing EBUS-TBNA. In one lung cancer suspected patient proposed for this procedure we identified a bronchial foreign body extracted with rigid bronchoscopy in another session.

EBUS-TBNA is currently used for preoperative lymph node staging of lung cancer, post-operative evaluation, diagnosis of centrally located intrapulmonary tumors or mediastinal and hilar adenopathy [9]. Being less invasive, safer than and as accurate as surgical staging in lung cancer patients, EBUS-TBNA is considered the test of first choice to confirm mediastinal lymph node involvement [10]. This procedure is generally performed under general anaesthesia to allow for a more precise ultrasound probe movement and needle position [11].

Our anaesthetic protocol consisted of propofol TIVA-TCI Schnider model aiming during induction for $\mathrm{Ce}=4.0-6.0 \mu \mathrm{g} / \mathrm{ml}$ according to patients ASA status. During the maintenance, an adequate level of hypnosis (BIS between 40 and 60) was obtained with a mean propofol $\mathrm{Ce}=3.1(+/-0.6) \mu \mathrm{g} / \mathrm{ml}$. In these circumstances hemodynamic parameters heart rates and mean arterial pressures had no major variation being $+/-10-20 \%$ from preoperative values.

The choice of anaesthetic technique and anaesthesia depth are continuous challenges for anaesthetists. Total Intravenous Anaesthesia is the preferred technique in an open circuit circumstances avoiding environment pollution. During inhalational anaesthesia, end tidal anaesthetic concentration correlates with effect site (brain) concentration and guides variation of hypnosis depths. By contrast, TIVA with propofol at a constant infusion rate results in an unpredictable and often suboptimal site effect concentration. We used in our patients propofol target controlled infusion. The major advantage of this technique is the continuous automatic adjustment of infusion rate in order to maintain the estimated plasma/effect site concentration equal to the set level. Despite the improved efficacy and safety profile of TIVA-TCI, propofol cerebral concentration is just estimated (not measured) according to a presumed pharmacokinetic models. In this respect, we used hypnosis monitoring with BIS as a component of our anaesthetic protocol. This approach is supported by the last UK National Audit Project (NAP5) on Accidental Awareness during General Anaesthesia in the United Kingdom and Ireland recommending BIS monitoring in all TIVA with muscle relaxants patients [12].

We varied propofol Ce (more than 2 $\mu \mathrm{g} / \mathrm{ml}$ ) in order to maintain BIS in a range of 40-60, aiming to avoid intra-anaesthetic awareness and to optimize anaesthetic depth according to procedural phases.

Currently available TIVA-TCI pumps use 2 pharmacokinetic models - Marsh and Schnider. The Marsh model assumes that the central compartment volume is directly proportional to weight only. The age is entered, but not used in the calculations, resulting in lack of accuracy. In Schnider model, lean body mass (estimated according to height and weight) and age are used to calculate doses and infusion rates resulting in better accuracy. For this reason, Schnider model results in lower propofol doses and should be preferred in elderly patients [13].

In our case series, the diagnosis or staging procedures were scheduled in lung cancer patients with reduced respiratory reserves. Our anaesthetic technique using propofol $\mathrm{TCl} \mathrm{Ce}$ ensures fast recovery without residual sedation.

Muscle relaxants were used in all our patients. For flexible bronchoscopy with EBUS-TBNA the use of LMA affords easy bronchial tree access, optimal operator comfort and avoids endotracheal intubation. At 
the end of procedure rocuronium was reversed with sugammadex, a very efficient antagonist fulfilling the requirements for fast recovery.

Another advantage of TIVA with propofol is the lower interference with the immune system. Along with the ongoing debate regarding the effect of anaesthetic and analgetic drugs on malignancy recurrence, reported data tend to support the use of propofol based anaesthesia due to a lesser immune suppressive effect [14-16].

In the present case series we used for the first time the recently introduced jet ventilation - SHFJV. During conventional ventilation, tidal volumes exceed anatomical and equipment dead space and gas exchange is largely related to bulk flow of gas to the alveoli. In high frequency ventilation the tidal volumes are smaller than total dead space. Therefore, bulk flow only partially contribute to gas exchange along with alternative mechanisms: pendelluft - redistribution of inspired gas from full, fast-filling units to slower-filling lung units, convective streaming or Taylor dispersion diffusion of the molecules with high axial velocities from central to lateral zones and cardiogenic mixing - air agitation in surrounding lung tissue by the heart beats [1720]. The limits of high frequency ventilation are related to the open ventilation system - lack of $\mathrm{CO}_{2}$ removal, airway pressure monitoring, tidal volume control and gas dryness.

By superposition of the normal frequency on high frequency ventilation, SHFJV increases minute ventilation and allows $\mathrm{CO}_{2}$ removal [21]. It may also increase positive end expiratory pressure and end-expiratory volume improving oxygenation [22], with low risk of barotrauma and air trapping. In an animal model, end-expiratory and tidal volumes determined by opto-electronic plethysmography were greater and gas exchange was improved with this technique [23]. Another advantage is lung protection as a result of low tidal volume ventilation and relative chest immobility.

In our patients, in order to improve oxygenation, we increased the inspiratory oxygen fraction, NF and HF ventilation emission pressure, the frequency of $\mathrm{HF}$ and the I:E ratio. The $\mathrm{PaCO}_{2}$ was maintained by increasing emission pressure and frequency of NF ventilation or by decreasing the frequency and I:E ratio of $\mathrm{HF}$ ventilation. Using a variable $\mathrm{FiO}_{2}=0.5-1$ and frequency on $\mathrm{HF}=400$ $550 / \mathrm{min}, \mathrm{SpO}_{2}$ was maintained stable with a mean intra-anaesthetic value of $98.5 \%$. $\mathrm{EtCO}_{2}$, intermittently measured every 5 minutes, had a mean value of $32(+/-5.4) \mathrm{mmHg}$, using a respiratory rate on $\mathrm{NF}=10-14 / \mathrm{min}$. Our results confirm other published data showing normocapnia in SHFJV patients [24].

Recorded airway pressures during the jet ventilation - PIP, MAP, PEEP - varied according to the patient pre-existent lung pathology and procedural particularities. We set a safety upper limit for the peak airway pressure of $25 \mathrm{mmHg}$ in order to avoid barotrauma. The Twin Stream ventilator, an electronic controlled multimode high frequency ventilator with two jet plateau, is simple to operate, has a quick setup and an intuitive user interface. Automatic initial settings calculated by body weight, extended monitoring capabilities including airway pressure monitoring and automatic manoeuvre for etCO $\mathrm{C}_{2}$ measurement, reduced risk of barotrauma with alarm pressure and stop ventilation, are greatest advantages. We recorded no intra-anaesthetic or postinterventional complications.

\section{Conclusion}

In order to circumvent challenges associated with diagnostic or interventional bronchoscopy in cancer patients we developed and applied an anaesthetic protocol in combination with Superimposed High Frequency Jet Ventilation. Our protocol enables a fast induction - fast recovery anaesthesia, cardiovascular stability and lowest immune suppression. SHFJV is a safe and effective method of ventilation in open system, maintaining good oxygenation and adequate $\mathrm{CO}_{2}$ removal and is highly suitable for endoscopic interventions. The results of our case series analysis point towards the efficacy and safety of these techniques, operator comfort and facilitation of the procedure. 


\section{Disclosure}

The authors have no conflict of interests to declare.

\section{References}

1. Pathak V, Welsby I, Mahmood K, Wahidi M, Maclntyre N, Shofe S. Ventilation and Anesthetic Approaches for Rigid Bronchoscopy. Ann Am Thorac Soc 2014; 11:628-634.

2. Sanders RD. Two ventilating attachments for bronchoscopes. Del Med J 1967; 39:170-175.

3. Giunta F, Chiaranda M, Manani G, Giron GP. Clinical uses of high frequency jet ventilation in anaesthesia. $\mathrm{Br} J$ Anaesth 1989; 63:102S-106S.

4. Fernandez-Bustamante A, Ibanez V, Alfara $J$, et al. High-frequency jet ventilation in interventional bronchoscopy: factors with predictive value on highfrequency jet ventilation complications. $J$ Clin Anesth 2006; 18:349-356.

5. Hautmann $H$, Gamarra $F$, Henke $M$, Diehm S, Huber RM. High freqency jet ventilation in interventional fiberoptic bronchoscopy. Anesth Analg 2000; 90:1436-1440.

6. Aloy A, Schachner M, Spiss CK, Cancura W. Tube-free translaryngeal superposed jet ventilation. Anaesthesist 1990; 39:493498.

7. Rezaie-Majd A, Bigenzahn W, Denk DM, et al. Superimposed high-frequency jet ventilation (SHFJV) for endoscopic laryngotracheal surgery in more than 1500 patients. Br J Anaesth 2006; 96(5):650659.

8. Smith I, Kranke $\mathrm{P}$, Murat $\mathrm{I}$, et al. Perioperative fasting in adults and children: guidelines from the European Society of Anaesthesiology. Eur $J$ Anaesthesiol 2011; 28:556-569.

9. Silvestri GA, Gonzalez AV, Jantz MA, et al. Methods for Staging Non-small Cell Lung Cancer Diagnosis and Management of Lung Cancer, $3^{\text {rd }}$ ed: American College

\section{Acknowledgement}

This paper was published under the frame of European Social Found, Human Resources Development Operational Program 2007-2013, project no. POSDRU/159/1.5/S/133377.

of Chest Physicians Evidence-Based Clinical Practice Guidelines CHEST 2013; 143(5) (Suppl):e211S-e250S.

10. Czarnecka K, Yasufuku K. Endobronchial Ultrasound-guided Transbronchial Needle Aspiration for Staging Patients with Lung Cancer with Clinical N0 Disease. Ann Am Thorac Soc 2015; 12(3): 297-299.

11. Pawlowski J. Purpose Anesthetic considerations for interventional pulmonary procedures. Curr Opin Anesthesiol 2013, 26:6-12.

12. NaidooD. Target Controlled Infusion. 2011.

[http://www.anaesthetics.ukzn.ac.za/Librari es/Documents2011/D_Naidoo_Target_Co ntrolled_Infusion.sflb.ashx available at 25.09.2015].

13. Pandit JJ, Cook TM. $5^{\text {th }}$ National Audit Project of The Royal College of Anaesthetists and the Association of Anaesthetists of Great Britain and Ireland. Accidental Awareness during General Anaesthesia in the United Kingdom and Ireland. Report and findings. 2015

14. Kurosawa $S$. Anesthesia in patients with cancer disorders. Curr Opin Anesthesiol 2012, 25:376-384.

15. Snyder GL, Greenberg S. Effect of anaesthetic technique and other perioperative factors on cancer recurrence. $\mathrm{Br} J$ Anaesth 2010; 105(2):106-115.

16. Tavare AN, Perry NJS, Benzonana LL, Takata $\mathrm{M}$ and Ma D. Cancer recurrence after surgery: direct and indirect effects of anesthetic agents. Int. J. Cancer 2012; 130:1237-1250.

17. Conlon C.E. High Frequency Jet Ventilation Anaesthesia Tutorial of the Week 271.2012. [https://www.aagbi.org/sites/default/files/27 
1\%20High\%20frequency\%20Jet\%20Ventil ation\%5B1\%5D.pdf available at 25.09.2015]

18. Chang HK. Mechanisms of gas transport during ventilation by high-frequency oscillation. J Appl Physiol 1984; 56:553563.

19. Rossing TH, Slutsky AS, Lehr JL, Drinker PA, Kamm R, Drazen JM. Tidal volume and frequency dependence of carbon dioxide elimination by high-frequency ventilation. N Engl J Med 1981; 305:13751379.

20. Fredberg JJ. Augmented diffusion in the airways can support pulmonary gas exchange. J Appl Physiol 1980; 49:232238.

21. Bacher A, Pichler K, Aloy A. Supraglottic combined frequency jet ventilation versus subglottic monofrequent jet ventilation in patients undergoing microlaryngeal surgery. Anesth Analg 2000; 90:460-465.

22. Kraincuk $P$, Kormoczi $G$, Prokop $M$, Ihra G, Aloy A. Alveolar recruitment of atelectasis under combined highfrequency jet ventilation: a computed tomography study. Intensive Care Med 2003; 29:1265-1272.

23. Sutterlin $R$, Priori $R$, Larsson $A$, LoMauro A, Frykholm P, Aliverti A. Frequency dependence of lung volume changes during superimposed high-frequency jet ventilation andhigh-frequency jet ventilation. Br J Anaesth 2014; 112(1):4149.

24. Koller-Halmer G, Schindler I, Koller $H$. SHFJV (Superimposed High Frequency Jet Ventilation), ETCO2 correlation to $\mathrm{PaCO}_{2}$ in diagnostic and therapeutic rigid bronchoscopy. Eur Respir J 2011; 38:s55. 